Malheureusement, rien n'est plus facile que de falsifier du lait de bufflesse avec du lait de vache, et, bien entendu, les éleveurs et les laitiers ne manquent pas de le faire : "Le lait de vache, dit MAYMone, peut être adjoint à un lait de bufflesse assez riche en graisse, en quantité voulue pour abaisser le taux de cette matière jusqu'à la teneur minimum assignée par la loi au lait de bufflesse $(6 \%)$ sans que l'analyse - en se basant sur les propriétés physiques et chimiques puisse sûrement révéler la fraude.

"Papper et Hogan ont recouru à la méthode biologique des précipitations spécifiques, obtenant des résultats assez satisfaisants. Mais cette méthode exige une technique de laboratoire qui ne se prête guère aux applications dans la pratique industrielle. ")

(A suivre).

\title{
A PROPOS DE LA THÉORIE DE LA DÉSACIDIFICATION ÉLECTRIQUE DU LAIT
}

\author{
par \\ le Professeur Dr Otтo GRATK
}

La revue "Le Lait", dans son numéro 151 de janvier 1936, a publié, sous le titre "A propos de la désacidification électrique du lait ", une étude de MM. Jean Pien, directeur, et Jacques BAIsse, ingénieur chimiste, des laboratoires des Fermiers Réunis.

Dans cette étude, les auteurs donnent une nouvelle explication des phénomènes, des réactions qui se produisent pendant la désacidification électrique du lait.

Ils contestent que la désacidification soit due à une décomposition de l'acide lactique par électrolyse et affirment qu'elle résulte de ce fait que les bases alcalino-terreuses sont mises en liberté - aux dépens surtout des phosphates lactiques et même des caséinates de phosphore - et viennent neutraliser l'acide lactique et une partie de la caséine.

C'est à ces mêmes bases libérées qu'il faudrait attribuer le fait que l'on peut rendre basique un lait traité par électrolyse et qui ne contient done plus d'acide lactique.

D'après la conception de MM. PIEn et BAISSE, la désacidification électrique du lait reposerait surtout sur la transformation électrolytique en phosphates d'aluminium des phosphates existants.

A cette théorie - que les auteurs eux-mêmes présentent d'ailleurs à titre hypothétique - on peut opposer ce qui suit :

Des essais, dont les résultats n'ont pas été publiés jusqu'à présent, mais ont été confirmés par des milieux compétents, ont prouvé que la désacidification électrique du lait pouvait parfaitement être 
obtenue, en utilisant des électrodes en charbon ou en platine qui ne pourraient évidemment donner lieu à aucune formation de phosphates d'aluminium. La décomposition de l'acide lactique, quelle que soit la matière constituante des électrodes, pourrait, d'ailleurs, être établie par une analyse de gaz, en récoltant séparément dans un électrolyseur spécial les différents gaz obtenus.

Les expériences indiquées ci-dessus montrent clairement que la théorie de MM. PIen et BaIsse ne peut guère être soutenue.

Il s'ensuit, au surplus, que la théorie établie par des chimistes attitrés et exposée dans mon article du No 132 de février 1934 de la revue Le Lait est probablement exacte, théorie qui conclut à la décomposition par électrolyse de l'acide lactique - aussi longtemps que celui-ci existe dans le lait et, en même temps, à une transformation en leur forme basique des sels acides du lait.

Aussi longtemps que nous en resterons réduits à de simples hypothèses sur la forme des sels organiques et inorganiques du lait, il est évidemment difficile d'émettre une conclusion absolument positive et on peut trouver d'autres théories, presqu'aussi plausibles, sur lesquelles toutefois, faute d'expériences suffisantes, je ne m'étendrai pas ici en ce moment.

Il est utile de mentionner encore qu'en employant des électrodes en charbon ou en platine, on peut rendre le lait basique tout aussi bien qu'avec des électrodes en aluminium.

On ne constate aucune odeur de chlore aux anodes, aucune trace de particules de charbon dans le lait, aucune réduction de poids des électrodes en charbon.

On a préféré, jusqu'à présent, les électrodes en aluminium parce que l'emploi du charbon entraînait certaines difficultés techniques qu'on surmontera tôt ou tard et pour la solution desquelles on dispose déjà d'éléments pleins de promesses. L'emploi du platine est parfaitement possible en théorie, mais il est exclu en pratique à cause du prix prohibitif du métal.

\section{REVUE}

\section{LE NETTOYAGE DES OBJETS ÉTAMIÉS EN LAITERIE}

\section{Emploi DU SUlfite DE SODIUM}

Les appareils et ustensiles utilisés en laiterie sont généralement en acier ou en cuivre, recouverts à chaud d'un revêtement d'étain. Le cuivre est en particulier un métal très employé, par suite de son prix relativement peu élevé, de la facilité avec laquelle on peut l'usiner et de sa conductivité thermique élevée. Toutefois, si le cuivre 